\title{
PCI-DDC Application Programming Interface: Performance in User-Level Messaging
}

\author{
Eric Renault, Pierre David, and Paul Feautrier \\ Laboratoire PRiSM \\ Université de Versailles - Saint-Quentin-en-Yvelines \\ 78035 Versailles Cedex, France \\ \{Eric.Renault, Pierre.David, Paul.Feautrier\}@prism.uvsq.fr
}

\begin{abstract}
Many programming interfaces which deal with peripherals (especially network peripherals) need to access the critical resources of the operating system, and system calls (or drivers) are generally used. Unfortunately, the time spent on such system calls is often greater than those requested for the operations themselves. In the case of the MPC machine, most of these operations do not need an intervention from the system and resources may be available from user applications. Our programming interface provides different levels of integration in the kernel depending on the security and the performance expected by the administrator. This article presents PAPI user-level performance.
\end{abstract}

\section{Introduction}

The MPC project started in 1995 as a collaborative endeavour between the LIP6 and PRiSM laboratories (France). Its goal is the development of both hardware and software layers to use the new HSL technology, a high speed network with an affordable price. An MPC machine is composed of nodes, each one with one or more processors and 1-Gbits/s links (HSL links). On each node, a FastHSL card is the interface between the network and the computer. An HSL link (IEEE 1355 [1]) is a bidirectional serial link which delivers a throughput of 1 Gbit/s. As 4 bits of control (generated by the crossbar to perform various controls) are transmitted with every byte, the effective bandwidth is limited to $80 \mathrm{MBytes} / \mathrm{s}$. The RCube (for Rapid Reconfigurable Router) component [2] is a $8 \times 8$ crossbar with a high bandwidth of 640 MBytes/s and a low latency of $150 \mathrm{~ns}$ per hop. Routing in this chip is wormhole and adaptative and it is possible to define different configuration tables for each of the 8 bidirectional links. The PCI-DDC (for PCI-Direct Deposit Component) component [3] is a network interface for message-passing multiprocessor architectures using HSL links and RCube routers. It is connected to the PCI bus (where it can act as a master) and sends/receives messages to/from the RCube component without processor intervention, using Direct Memory Access. A Fast-HSL card is a 32bit 33-MHz PCI card which includes the PCI-DDC and RCube components. It provides seven HSL ports, each one connected to a RCube entry; the last entry of the RCube chip is connected to the PCI-DDC component. In this article, we 
present a short description of the PCI-DDC component and a fast presentation of PAPI. Then, our user-level results are compared to those of Active Messages, BIP and Fast Messages.

\section{Programming the PCI-DDC Component}

The aim of the PCI-DDC component is to exchange messages with other nodes via the HSL network and the RCube components. Interface with the CPU uses two circular lists in main memory and some registers to store pointers in these lists. The two lists are the LPE (List of Pages to Emit) where CPU describes messages to send and the LMI (List of Message Identifiers) where the PCI-DDC component writes the description of each received message. A message identifier is a 24-bit integer which identifies a message; a single node does not permit two incoming messages with the same identifier at the same time.

The component allows the application to send two different message types: normal messages are using the "remote-write" protocol 44, i.e. both local and remote physical addresses must be specified by the sender; short messages do not need any physical address, i.e. instead of specifying local and remote physical addresses, the content of the message is placed directly in these fields. Short messages are particularly important during the initialization phase of an application, for example, since they are the only way to exchange physical addresses needed for normal messages. The size of these short messages is limited to 8 bytes, the room available in LPE and LMI entries.

PAPI [5], which stands for PCI-DDC Application Programming Interface, is a modular interface. It allows the system administrator to choose the security level of the system depending on the performance desired and the users. In the current version, three levels of integration are available:

- NONE: no security is provided. All of the module code is placed in user mode except some system calls (used only at initialization), used to access information from the kernel;

- ACCESS: traditional system security is provided, i.e. code is placed in kernel space and a system call is performed each time the application calls functions from our API;

- HIDE: the total security configuration, i.e. the code is placed in kernel space and no system call is provided to access it. The only way to run a function of a hidden module is to have another module with an ACCESS configuration, and system calls to access the functions of the hidden module.

\section{Performance}

The program used to determine the performance times of the MPC machine (see below) is the classical ping-pong program. It sends and receives one million messages between two nodes (one message can be sent if the message from the remote node has been received). Once this is done, the elapsed time is divided 
by two millions in order to determine the total average time to send one message from one node to another one (i.e. one-way latency). The effective user bandwidth is computed by dividing the size of messages by the latency (throughout this section, we will refer to the effective user bandwidth as bandwidth).

At the PRiSM laboratory, each node of our MPC machine is composed of two 233-MHz Pentium II with $32 \mathrm{MB}$ of memory. Another network (a 10-Mbit/s Ethernet TCP/IP network) is used for control operations, such as program launching. The operating system is FreeBSD 3.2. We will discuss our results by comparing them with Myrinet [6] message-passing libraries, especially Active Messages [7, BIP [8] and Fast Messages [9]. These support an architecture similar to that of the MPC project, i.e. a cluster of PCs, and are some of the fastest available. Performance results from these other message-passing libraries are taken from the literature [10]11] 12, and not measured directly.

Figure 1 shows the time required to send a message from one node to another for different message-passing libraries. It shows that a latency for message sizes less than or equal to 64 bytes is quite similar for BIP, Fast Messages and PAPI. However, PAPI has a far better latency for messages larger than 64 bytes.

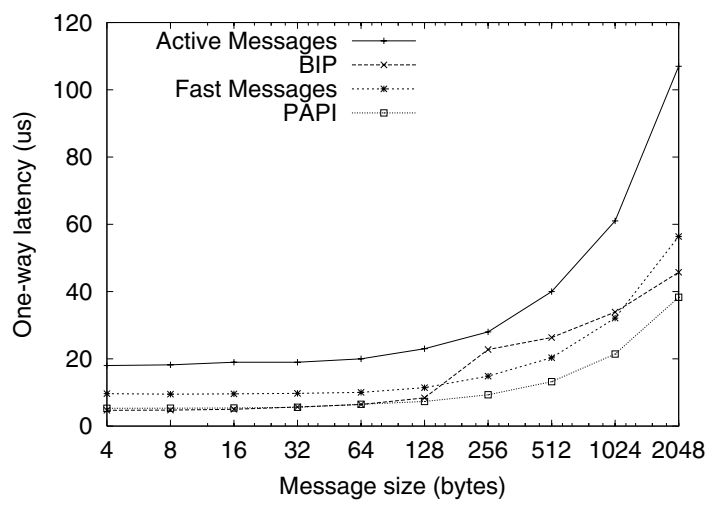

Fig. 1. One-way latency for different message-passing libraries

The anomaly in the BIP curve above 128 bytes (see Fig. 2) is due to the short message concept of BIP. The boundary is software-dependent, and reflects the threshold where messages are sent directly to the host memory rather than copied in the adapter memory. The PCI-DDC concept of short message is quite different: short message data are copied in the LMI of the receiving node, and are restricted to 8 bytes, the room available in LPE and LMI entries.

Where is the latency time spent? Figure 3 analyses the details of the transmission of a short message. On the sender node, the application calls our PAPI_SSend function, which fills an entry in the LPE in memory, performs a write in a PCI$\mathrm{DDC}$ register which is located in PCI configuration space, and returns. Once 


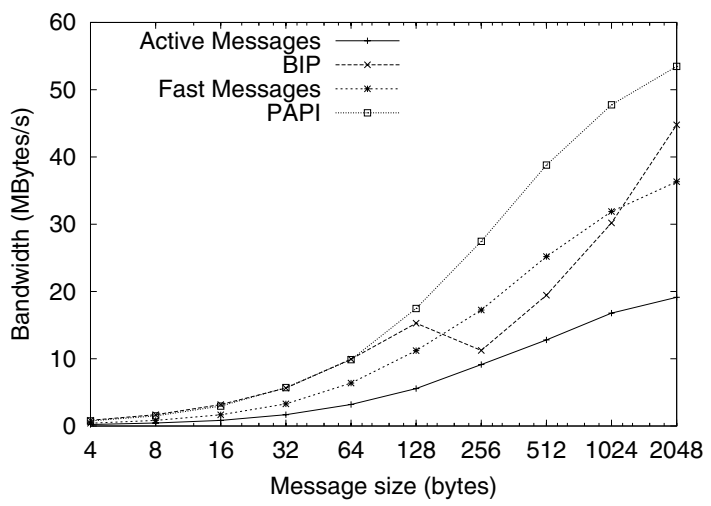

Fig. 2. Bandwidth for different message-passing libraries

informed by the write that a new message must be sent, the PCI-DDC component starts to work. Hardware specifications [3] [2] tell us that the components and the network spend $1.7 \mu \mathrm{s}$ (hardware latency) to bring the message to the other node memory. On the receiver node, where the PAPI_Receive function is called by the application, the LMI list is monitored. As soon as it is updated by the receiving PCI-DDC component, PAPI_Receive returns with information about the incoming message.

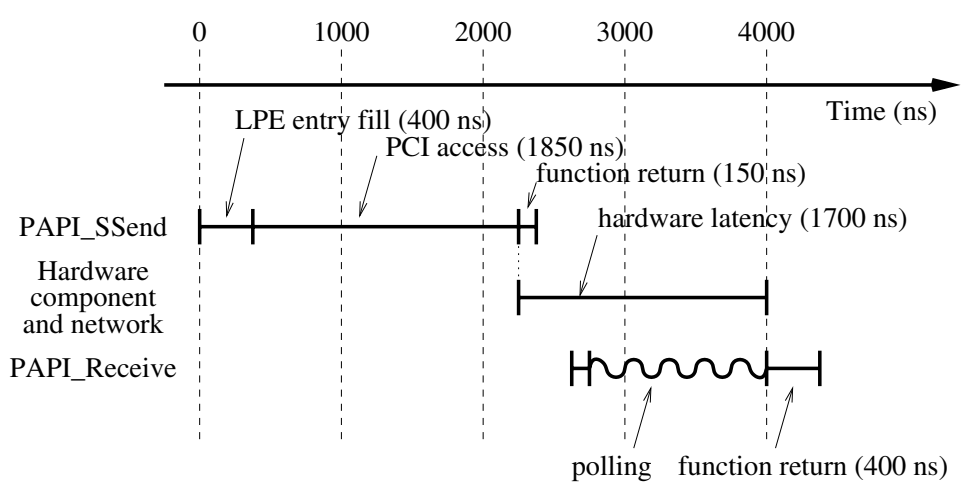

Fig. 3. Decomposition of the transmission time for a short message

This figure highlights another important point: during the transmission of the message, only a little part $(0.8 \mu$ s over $4.39 \mu$ s, i.e. less than $20 \%)$ depends upon the CPU speed. The rest of the time depends upon the PCI bus speed and the hardware latency. The greatest part (more than $80 \%$ ) of the latency time is 
thus independent of the CPU. So this means that PAPI is close to the minimum latency for the Fast-HSL card.

\section{Conclusion}

In this article we have presented PAPI, an application programming interface for the MPC machine. We have shown that, even for messages of less than one system page $(4 \mathrm{kB})$, our programming interface takes advantage of the HSL link bandwidth. The latency is very close to the minimum latency one can expect from the Fast-HSL cards.

Our study shows that a large part of the latency time is spent on a PCI access through a configuration space register. Given the same HSL network and router (RCube), improvements could be made only with modified access to the PCIDDC registers, through a memory-mapped access. However, this would require a modification of the PCI-DDC hardware component.

\section{References}

[1] C. Whitby-Strevens and al. IEEE Draft Std P1355 — Standard for Heterogeneous Interconnect - Low Cost Low Latency Scalable Serial Interconnect for Parallel System Construction, 1993.

[2] V. Reibaldi. RCube Specifications. Laboratoire Informatique de Paris VI, February 1997.

[3] J.J. Lecler, F. Potter, A. Greiner, J.L. Desbarbieux, and F. Wajsburt. PCI-DDC Specifications. Laboratoire Informatique de Paris VI, 1996.

[4] F. Potter. Conception et réalisation d'un réseau d'interconnexion à faible latence et haut débit pour machines multiprocesseurs. $\mathrm{PhD}$ thesis, Université Pierre et Marie Curie, 1996.

[5] E. Renault. Pci-ddc Application Programming Interface User Manual. Research report, Laboratoire PRiSM, Université de Versailles - Saint-Quentin, France, May 2000.

[6] N.J. Boden, D. Cohen, R.E. Flederman, A.E. Kulawik, C.L. Seitz, J.N. Selzovic, and W.-K. Su. Myrinet - A Gigabit-per-Second Local-Area Network. In IEEEMicro, volume 15, pages 29-36, 1995.

[7] T. von Eicken, D.E. Culler, S.C. Goldstein, and K.E. Schauser. Active Mesages: a Mechanism for Integrated Communication and Computation. In 19th International Symposium on Computer Architecture, 1992.

[8] L. Prylli. BIP Messages User Manual for BIP 0.94, June 1998.

[9] S. Pakin, V. Karamcheti, and A.A. Chien. Fast Message (FM): Efficient, Portable Communication for Workstation Clusters and Massively-Parallel Processors. In IEEE Concurrency, volume 5, pages 60-63, 1997.

[10] S. Araki, A. Bilas, C. Dubnicki, J. Edler, K. Konishi, and J. Philbin. UserSpace Communication: A Quantitative Study. In The SuperComputing, Orlando, Florida, 1998.

[11] Concurrent Systems Architecture Group. Fast Messages Performance. Web page, 1999. http://www-csag.ucsd.edu/projects/comm/fm-perf.html.

[12] High Speed Networks and Multimedia Application Support. BIP Performance Curves. Web page, 1997. http://lhpca.univ-lyon1.fr/Resultats/bipres.html. 